\title{
Walking gait event detection based on electromyography signals using artificial neural network
}

\begin{abstract}
In many gait applications, the focal events are the stance and swing phases. Although detecting gait events using electromyography signals will help the development of assistive devices such as exoskeleton, orthoses, and prostheses, stance and swing phases have yet to be observed using electromyography signals. The core of this study is to propose a classification system for both stance and swing phases based on electromyography signals. This is to be done by extracting the patterns of electromyography signals from time domain features and feeding them into an artificial neural network classifier. In addition, a different number of input features and two prominent training algorithm of artificial neural network have been employed in this study. Eight subjects that participated in this study were divided into two categories namely, learned (first seven subjects) and unlearned data (the remaining one subject). It was observed that Levenberg-Marquardt algorithm with five time domain features performed better than other features with an average percentage of classification accuracy of 87.4\%. This system was further tested with electromyography signals of learned and unlearned data to identify the stance and swing phases in order to detect the timing of heel strike and toe off. The mean absolute different values between artificial neural network and footswitch data for learned data were $16 \pm 18 \mathrm{~ms}$ and $21 \pm 18 \mathrm{~ms}$ for heel strike and toe off, respectively. For this case, no significant differences $(p<0.05)$ were observed in mean absolute different for heel strike and toe off detections. Besides, the mean absolute different values of unlearned data were shown to be acceptable, $35 \pm 25 \mathrm{~ms}$ for heel strike and $49 \pm 15 \mathrm{~ms}$ for toe off. By the end of this experiment, basing the examination of gait events with electromyography signals using artificial neural network is possible.
\end{abstract}

Keyword: EMG signals; Gait event; Artificial neural network; Time domain features 